\title{
Middle School Students' Perceptions of Culturally and Geographically Relevant Content in Physical Education
}

\author{
Luciana Braga (Corresponding author) \\ Department of Foundations \& Secondary Education, University of North Florida \\ 1, UNF Drive Jacksonville, FL 32224 United States \\ E-mail:1.braga@unf.edu \\ Eloise Elliott \\ College of Physical Activity and Sports Sciences, West Virginia University \\ PO Box 6116 Morgantown, WV 26506-6116 United States \\ E-mail: Eloise.Elliott@mail.wvu.edu \\ Emily Jones \\ College of Physical Activity and Sports Sciences, West Virginia University \\ PO Box 6116 Morgantown, WV 26506-6116 United States \\ E-mail: Emily.Jones@mail.wvu.edu \\ Sean Bulger \\ College of Physical Activity and Sports Sciences, West Virginia University \\ PO Box 6116 Morgantown, WV 26506-6116 United States \\ E-mail: Sean.Bulger@mail.wvu.edu
}

Received: 25-09- 2015

Accepted: 26-10- 2015

Published: 31-10- 2015

doi:10.7575/aiac.ijkss.v.3n.4p.62

URL: http://dx.doi.org/10.7575/aiac.ijkss.v.3n.4p.62

This study was funded by the United States Department of Education through the Carol M. White Physical Education Program (PEP) Grant.

\begin{abstract}
Introduction: Advocates for non-traditional approaches to physical education (PE) emphasize the need for physical educators to design curricula that foster students' engagement in physically active lifestyles outside of the school setting. Accordingly, current guidelines for PE curriculum design recommend the inclusion of content that is relevant to the students' cultural background and their geographical environment (Society of Health and Physical Educators, 2009, 2015). Purpose: This study investigated how less predisposed to be active (LPA) and more predisposed to be active (MPA) students perceived the incorporation of culturally and geographically relevant content in a newly developed standards-based middle school PE curriculum. Methods: The study followed a sequential mixed-methods approach. In Phase One, two instruments measured attraction to physical activity and perceived athletic competence of 116 students. In Phase Two, 47 students (selected based on Phase One results) participated across twelve focus groups. Results: Four overarching themes emerged from both LPA and MPA focus groups' data: (a) Enjoyment, (b) Learning, (c) Value, and (d) Challenges. Findings from this study revealed a sense of increased perceptions of competency and value of PE among students as a result of their engagement with the new content. Conclusion: This study underlines the importance of selecting PE content that is innovative, challenging, meaningful, and relevant to the students' culture and geographical environment.

Keywords: physical education, physical activity, curriculum, culture, geographical environment.

\section{Introduction}

The Society of Health and Physical Educators (SHAPE, 2015) has recently identified the following components as essential for the structure of quality physical education (PE) programs: (a) policy and environment, (b) curriculum, (c) appropriate instruction, and (d) student assessment. Although researchers have documented a number of significant barriers to effective PE programs in middle and high schools associated with policy and environment (i.e., insufficient allocated time, large class sizes, equipment shortage, inadequate facilities, and marginalization of the discipline (Bulger \& Housner, 2009; Prusak, Pennington, Graser, Beighle, \& Morgan, 2010) the adoption of traditional multi-activity curricula continues to be a hindrance for students' engagement (Bulger \& Housner, 2009; Ennis, 2014). Traditional multi-activity curricula, which are characterized by short units of instruction focused on team sports (Kirk, 2006), have been criticized in the literature and indicated as problematic for several reasons, the most important of which being that highly skilled "athletic" students dominate the activities at the expense of the participation of less skilled students
\end{abstract}


(Ennis, 2014; Garn, Cothran, \& Jenkins, 2011). Furthermore, when PE content is narrowed to team sports, students are prevented from developing the competencies and confidence needed to participate in alternative forms of physical activity (PA) that could be carried on for a lifetime (Ferry \& McCaughtry, 2013; SHAPE, 2014).

In response to the inadequacies inherent to traditional, multi-activity approaches to PE, a variety of alternative curriculum models have been successfully developed, researched, and disseminated in the United States and other countries (Ennis, 2006, 2014). Although advocates for non-traditional approaches to PE address the matter from different perspectives, there is strong consensus on the need for curricula that fosters PA engagement outside of school as a result of participation in PE. Physical education curricula in the United States should be based on the National Physical Education Content Standards (SHAPE, 2014) and on state and/or district guidelines, if available. Moreover and equally important, PE curriculum should be "culturally and geographically relevant to the needs of the population it serves" (SHAPE, 2009). This means that PE curriculum should include content that fits the contextual environment in which it is being delivered, reflecting and contributing to the popular PA culture (Enright \& O'Sullivan, 2012). Toward this end, scholars have emphasized the importance of embracing students' interests and cultural backgrounds as well their geographical environment in the process of curriculum development.

In order to design relevant and meaningful PE curricula educators must first gain understanding of students' unique personal and cultural perspectives about movement and PE. Because innovative approaches to PE aim to offer young people an element of choice, to acknowledge their interest, and to resonate with their cultural background, it is critical that curricular decisions account for their voices during planning, implementation, and evaluation phases (Enright \& O'Sullivan, 2013; Oliver \& Oesterreich, 2012). By listening and responding to youth's voices regarding movement experiences, physical educators will be better prepared to offer them appealing and realistic movement experiences (Tanehill, MacPhail, Walsh, \& Woods, 2015). Unfortunately, the most common research approach used to study youth's movement experiences is to conduct research "on children" as opposed to research "with or for children;" the former approach neglects the centrality of students individual and cultural perspectives about issues related to their wellbeing (Darbyshire, MacDougall, \& Schiller, 2005, p. 419). To date, purposeful and systematic assessment of students' responses to PE curricula in the United States remains scarce (Prusak, Davis, Pennington, \& Wilkinson, 2014). Oliver and Oesterreich (2012) argued that "one way to engage young people is to listen to them, value their voices and understand how to utilize their feedback in the creation and development of curriculum" (p.395). The purpose of this study was to examine middle school students' perceptions of the inclusion of culturally and geographically relevant content (CGRC) in the PE curriculum. Specifically, this study aimed at gaining understanding of how students who are more- and students who are less- predisposed to be physically active perceived the inclusion of CRGC content in a newly developed standards-based middle school PE curriculum.

\section{Method}

\subsection{Context}

This study was part of a larger, three-year school-based health intervention funded by the United States Department of Education Carol M. White Physical Education Program (PEP) grant and implemented in the two middle schools of an Appalachian district. The project, titled CHOICES (Children's Health Opportunities Involving Coordinated Efforts in Schools) consisted of an integrated approach aimed at adolescent health improvement using three complementary components: schools, health care, and community. The school component of CHOICES focused on the establishment of a Comprehensive School Physical Activity Program with an emphasis on: (a) the development of a standards-based PE curriculum guide, (b) continuous professional development for PE teachers, (c) before and after school PA programming, and (d) opportunities for healthy eating.

2.1.1 Middle school standards-based physical education curriculum guide

Prior to the CHOICES project both middle schools did not have or follow a specific PE curriculum. Over a three-year period, PE teachers engaged in a professional development initiative designed to empower them to develop a middle school PE curriculum guide. Throughout multiple professional development workshops, PE teachers learned new content, wrote objectives, designed scope and sequences, developed instructional strategies, and selected assessments for each unit of instruction. During this process, content experts were brought to the school, equipment was purchased, and teachers worked collaboratively to write a curriculum that includes 12 units $(5$ team/dual sports; 7 individual activities). As units became ready, they were gradually implemented in both schools and at the completion of the threeyear project the curriculum guide was submitted for external review based on the PECAT (Centers for Disease Control and Prevention, 2006). According to the external reviewer the curriculum was recognized as consistent with the national and state standards and included all the components required for the delivery of quality PE at the middle school level.

2.1.2 Culturally and geographically relevant content

In this study, CGRC is defined as curricular units of instruction that differ from traditional PE content (i.e., team-sports, exercise routines) and have meaning to the environment in which the students live, learn, and play and is determined by the culture of their families, the physical environment surrounding them, and what resonates with their way of life. Prior to the start of the project, 645 students from both schools were asked to answer an electronic Student Physical Activity Interest Survey and rate a wide variety of PAs and sports on a four-point scale ranging from no way to so cool. Equipped with the survey results, the curriculum development team deliberated about the feasibility of including the selected content in the curriculum. Factors that were considered in this discussion included: (a) students' preferences, (b) the relevance of the content to the Appalachian community in which the schools are located, (c) the availability of resources and partnerships within the schools (i.e., facilities and outdoor environment) and within the community (i.e., built and natural environment, content experts, local business for equipment purchase and maintenance), and (d) the 
potential leadership roles that PE teachers could take regarding each unit development. Examples of CGRC included in the curriculum guide are: archery, mountain biking, slacklining, rock climbing, rhythmic activities, and disc golf. All these activities are aligned with students' interests and were rated as looks like fun or so cool by at least $80 \%$ of the students in both schools. Not only do these activities represent the PA culture of the Appalachian community in which the schools are located, they are appropriate to the geography of the location and the facilities available to local residents there. This study focused on students' perceptions of mountain biking, archery, and slacklining because at the time of data collection they had been exposed to full units of these activities in the respective PE classes.

2.2 Setting

This study was conducted in one of the schools served by the CHOICES project. Approximately one third of all middle school-aged children in the district attend this school, which will be referred as Western School. In the year this study was conducted enrollment in Western School was 312 students (169 female and 143 male). Sixty seven percent of the students are qualified for free or reduced lunch. The predominant ethnicity in the Western school is white accounting for $96.2 \%$ of the students, while black and other ethnicities account for $3.8 \%$ of the student body. Two physical educators ( 1 male and 1 female) implemented the new units at Western School.

\subsection{Research Design}

This study employed a sequential mixed methods design. In Phase One, two questionnaires were used to collect data related to middle school students' perceived athletic competence and attraction to PA. This information was used to identify two distinct groups of students (those who are more- and those who are less- predisposed to be physically active) to take part in focus groups interviews in Phase Two. The Youth Physical Activity Promotion (YPAP) model (Welk, 1999) was the theoretical framework used as the foundation for the selection of these two groups of participants. The YPAP model acknowledges that multiple personal, social, and environmental factors influence youth PA behavior and classify those factors into enabling, predisposing, reinforcing, and personal demographics (Welk, 1999) (See Figure 1). At the core of the YPAP model are predisposing factors, which include psychological variables that have been indicated by research to enhance the likelihood that a child or adolescent will regularly participate in PA (Welk, 1999). In the YPAP model, predisposing factors are summarized into two questions: (a) Am I able? and (b) Is it worth it? The first question addresses the individuals' perceptions of his or her capabilities to perform in sports and PAs while the second question addresses the person's expectancy regarding the outcomes of engaging in sports and PAs (Welk \& Schaben, 2004). According to Welk (1999) "youth who can answer yes to both questions will likely possess an 'active identity' and be predisposed to an active lifestyle" (p.13).

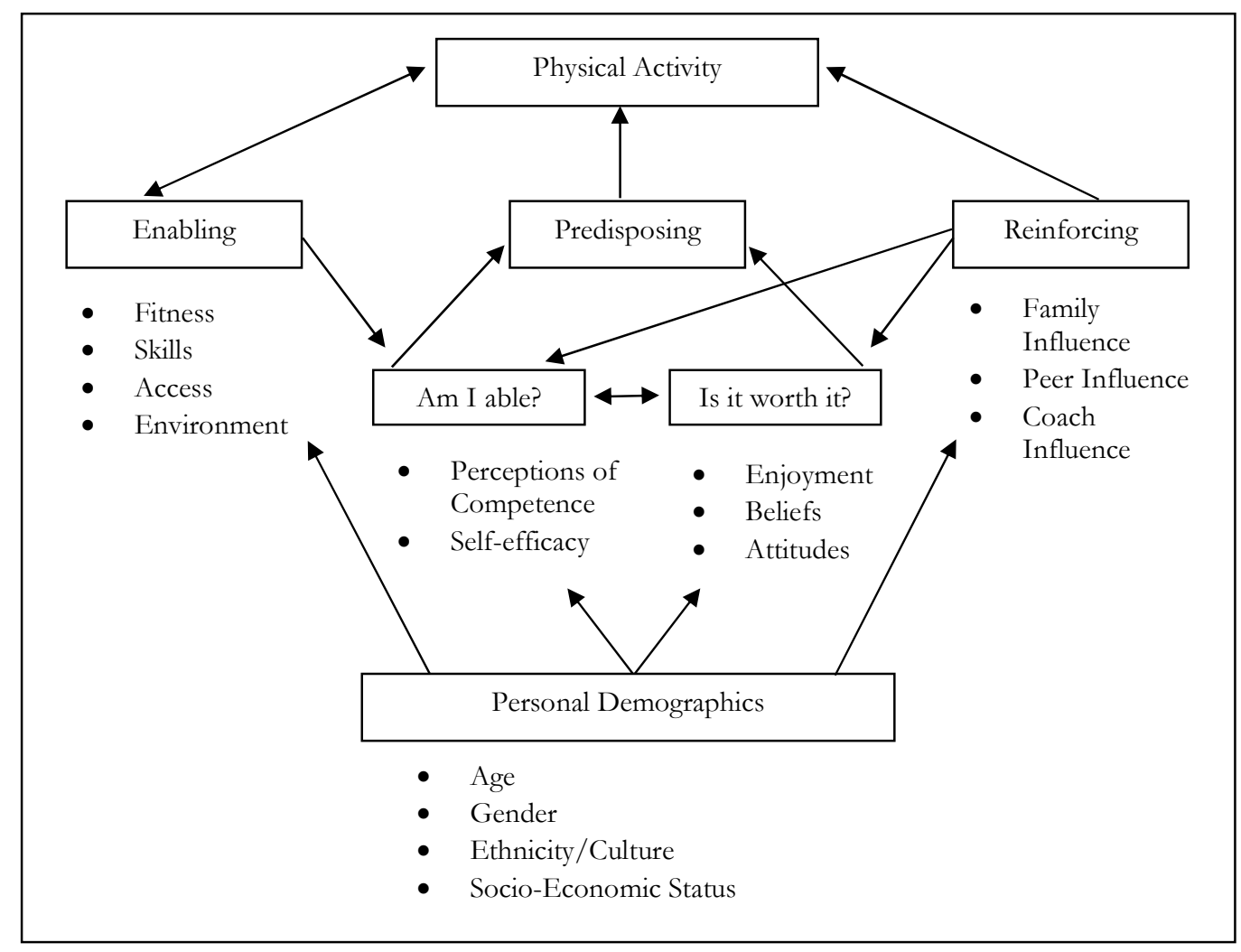

Figure 1. The Youth Physical Activity Promotion Model (Welk, 1999)

\subsection{Phase One: Determining Students' Predispositions to be Physically Active}

\subsubsection{Participants}

All sixth, seventh, and eighth grade students who were enrolled in PE, and who had not opted out of the CHOICES study were invited to participate in Phase One. On data collection day, 120 middle school students completed Phase 
One questionnaires but four participants were deleted listwise because of missing data. The total number of participants in Phase One was 116 students (53 male; 63 female) ranging in age from 11 to 15 years old $(M=12.3 ; S D=1.54)$.

\subsubsection{Data sources}

Two questionnaires were used in Phase One to measure middle school students' perceived athletic competence and attraction to PA.

Athletic competence subscale of the Self-Perception Profile for Children. The athletic competence subscale of the SelfPerception Profile for Children (SPPC) scale was used to measure students' perceptions of their ability to successfully participate in sports and PAs (Harter, 2012). The SPPC scale follows a structured alternative format, in which each item presents children with two statements for them to determine which one best represents them and then choose if the statement is sort of true or really true for them. Items of the SPPC scale are scored from 1 to 4, being the highest score corresponded to the answer that represents the most athletic self-evaluation (Harter, 2012). The subscale has six items and individual scores are obtained averaging all items' score. Previous studies have found satisfactory internal reliability $(\alpha>.70)$ within the athletic competence subscale of the SPPC scale across different samples (Baker \& Davison, 2011; Harter, 2012; Muris, Meesters, \& Fijen, 2003).

Children's Attraction to Physical Activity scale. The short version of the Children's Attraction to Physical Activity (CAPA) scale was used to measure students' emotional responses to their involvement in sports and PAs (Brustad, 1993). Similar to the SPPC scale, the CAPA scale follows a structured alternative format and items are scored from 1 to 4 , being the highest score corresponded to the answer that shows the most compelling attraction to PA. The scale has 15 items and individual scores are obtained by averaging all the items' scores. The CAPA scale has also been indicated to have satisfactory internal reliability $(\alpha>$.70) across different samples (Brustad, 1993, 1996; Paxton, Estabrooks, \& Dzewaltowski, 2004).

\subsubsection{Data Analysis}

The following analyses were conducted for the entire sample and for each grade level sample independently: (a) descriptive statistics and internal consistency reliability analysis using Cronbach's Alpha for the CAPA scale, the SPPC's athletic competence subscale, and for both scales combined, (b) distribution tertiles for the both scales combined, and (d) descriptive statistics for the lower and upper tertiles of both scales combined.

\subsection{Phase Two: Exploring Students' Perceptions of CGRC in PE}

\subsubsection{Participants}

A theory-based sampling strategy (Marshall \& Rossman, 2006), stratified by grade was used to select participants according to their potential to represent the predisposing factors included in the YPAP model (Welk, 1999). Potential participants for Phase Two were identified based on Phase One data analysis and results. Students who had the 33\% lowest mean scores in Phase One were invited to participate in Phase Two and were called less predisposed to be active $(\boldsymbol{L P} A)$. Students who had the 33\% highest mean scores in Phase One were also invited to participate in Phase Two and were called more predisposed to be active (MPA). A total of 76 students across sixth, seventh, and eighth grades were invited to participate in Phase Two (38 LPA; $38 \mathrm{MPA}$ ) and 57 of them returned the signed consent letters. Because ten students were not available to take part in the focus groups the day they were conducted, Phase Two participants were 47 students (21 LPA, 26 MPA; 17 male, 30 female) in grades sixth, seventh, and eight, ranging in age from 11 to 15 years old.

\subsubsection{Data sources}

Focus groups interviews were used in Phase Two given their potential to encourage participants to express a variety of viewpoints in a safe atmosphere through group interactions (Krueger \& Cassey, 2009). A total of 12 focus groups were conducted (Seven groups composed of LPA students and five groups composed of MPA students) with an average of four participants per focus group. Each focus group followed a semi-structured questioning format and lasted, in average 21 minutes. All focus groups were conducted during regular school hours over two consecutive days. The primary researcher and a research assistant were the focus groups facilitators.

\subsubsection{Data analysis}

Phase two of this study employed an inductive content analysis. Specifically, a three-phase approach suggested by Miles and Huberman (1994) was used and involved the following actions: (a) data reduction, (b) data display, and (c) conclusion drawing and verification. Throughout this process, segments of data with similar meanings were identified, coded, and organized into categories reflecting related patterns also identified as themes. The coded segments of data and the categories of shared meaning were often revisited through a constant comparative approach with the purpose of examining their consistency and relatedness (Glaser \& Strauss, 1967). In order to establish trustworthiness of the analysis, different researchers coded segments of data independently and, through multiple debriefing meetings variances in coding were discussed and categories further defined to ensure a shared valuation system and consistency across the coding schemes. 


\section{Results}

\subsection{Phase One}

The internal consistency reliability analysis showed that Cronbach's Alphas for both scales combined were equal to or higher than the Cronbach's Alpha for each individual scale across the entire sample and in each grade level sample (See Table 1). Therefore, participants' mean scores were computed by averaging the total 21 items from both the athletic competence subscale of the SPPC scale and the CAPA scale. Subsequent data analyses were based on participants' mean scores for both scales combined. The average scores for scales combined in the entire sample was $M=2.98, S D$ $=.60$ (range 1-4). Although scores were relatively high in all grade levels, they were higher in seventh grade $(M=3.15$, $S D=.61)$, than either in eighth grade $(M=3.06, S D=.54)$, or sixth grade $(M=2.81, S D=.61)$ (See Table 2$)$. Mean scores distributions were slightly negatively skewed in all grade levels, indicating that participants generally reported (on a 4 point scale) high degrees of perceived athletic competence and attraction to PA. Hence, in order to obtain the two distinct groups of participants for Phase Two (LPA \& MPA), the mean scores' distribution in each grade was divided into tertiles: the lower 33\% scores, the middle 33\% scores, and the upper 33\% scores (See Table 3).

Table 1. Phase One: Instruments’ Reliability Coefficients (Cronbach’s Alpha)

\begin{tabular}{cccc}
\hline Grade & $\begin{array}{c}\text { SPPC scale (athletic } \\
\text { competence subscale) }\end{array}$ & CAPA scale & Both scales combined \\
\hline $6^{\text {th }}$ & .68 & .89 & .91 \\
$7^{\text {th }}$ & .72 & .91 & .92 \\
$8^{\text {th }}$ & .52 & .90 & .90 \\
All & .65 & .90 & .91 \\
\hline
\end{tabular}

Table 2. Phase One: Mean Scores (M) and Standard Deviations (SD)

\begin{tabular}{|c|c|c|c|c|c|c|}
\hline \multirow[t]{2}{*}{ Grade } & \multicolumn{2}{|c|}{$\begin{array}{l}\text { SPPC scale (athletic } \\
\text { competence subscale) }\end{array}$} & \multicolumn{2}{|c|}{ CAPA scale } & \multicolumn{2}{|c|}{ Both scales combined } \\
\hline & $M$ & $S D$ & $M$ & $S D$ & $M$ & $S D$ \\
\hline $6^{\text {th }}$ & 2.74 & .65 & 2.84 & .65 & 2.81 & .61 \\
\hline $7^{\text {th }}$ & 3.06 & .67 & 3.18 & .65 & 3.15 & .61 \\
\hline $8^{\text {th }}$ & 2.95 & .54 & 3.10 & .63 & 3.06 & .57 \\
\hline All & 2.89 & .63 & 3.02 & .65 & 2.98 & .60 \\
\hline
\end{tabular}

Table 3. Phase One: Lower and Upper Tertiles for Both Scales Combined

\begin{tabular}{ccccccc}
\hline Grade & \multicolumn{3}{c}{ Lower 33\% } & \multicolumn{3}{c}{ Upper 33\% } \\
\hline & Range & $M$ & $S D$ & Range & $M$ & $S D$ \\
\cline { 2 - 7 } $6^{\text {th }}$ & $1.38-2.39$ & 2.12 & .28 & $3.24-3.86$ & 3.53 & .16 \\
$7^{\text {th }}$ & $2.00-2.81$ & 2.40 & .28 & $3.57-4.00$ & 3.78 & .16 \\
$8^{\text {th }}$ & $1.62-2.81$ & 2.43 & .39 & $3.38-3.81$ & 3.62 & .13 \\
All & $1.38-2.67$ & 2.27 & .32 & $3.38-4.00$ & 3.62 & .17 \\
\hline
\end{tabular}

\subsection{Phase Two}

As a result of the inductive content analysis, four themes emerged from both LPA and MPA focus groups data: (1) Enjoyment, (2) Learning, (3) Value, and (4) Challenges. Although findings indicated that LPA and MPA students had similar perceptions regarding the content, a few differences in the frequencies of comments amongst the two groups were observed (See Table 4). 
Table 4. Phase Two: Frequencies of LPA and MPA Students' Comments

\begin{tabular}{|c|c|c|c|c|}
\hline \multirow[t]{2}{*}{ Themes \& Subthemes } & \multicolumn{2}{|c|}{ LPA } & \multicolumn{2}{|c|}{ MPA } \\
\hline & Comments & Percentage & Comments & Percentage \\
\hline I. Enjoyment & 223 & $35.6 \%$ & 198 & $39.2 \%$ \\
\hline New opportunities & 39 & $6.2 \%$ & 39 & $7.7 \%$ \\
\hline Fun, cool, \& exciting content & 104 & $16.6 \%$ & 96 & $19 \%$ \\
\hline Activities attributes & 80 & $12.8 \%$ & 63 & $12.5 \%$ \\
\hline II. Learning & 137 & $21.9 \%$ & 117 & $23.1 \%$ \\
\hline Skills and concepts & 41 & $6.5 \%$ & 43 & $8.5 \%$ \\
\hline Successful experiences & 65 & $10.4 \%$ & 39 & $7.7 \%$ \\
\hline Social support & 31 & $5 \%$ & 35 & $6.9 \%$ \\
\hline III. Value & 140 & $22.4 \%$ & 125 & $24.9 \%$ \\
\hline Sociocultural relevance & 35 & $5.6 \%$ & 36 & $7.2 \%$ \\
\hline Better than traditional PE & 69 & $11 \%$ & 53 & $10.5 \%$ \\
\hline More learning opportunities & 36 & $5.8 \%$ & 36 & $7.2 \%$ \\
\hline IV. Challenges & 126 & $20.1 \%$ & 64 & $12.8 \%$ \\
\hline Safety & 44 & $7 \%$ & 36 & $7.2 \%$ \\
\hline Equipment differentiation & 33 & $5.3 \%$ & 10 & $2 \%$ \\
\hline Uncomfortable experiences & 49 & $7.8 \%$ & 18 & $3.6 \%$ \\
\hline Total & 626 & $100 \%$ & 504 & $100 \%$ \\
\hline
\end{tabular}

\subsubsection{Theme I: Enjoyment}

Students across all grade levels consistently reported enjoying being exposed to the CGRC. Several LPA and MPA students mentioned being "excited" with the "opportunity to learn" "new," "different," and "unusual" activities. Data revealed a strong sense of appreciation among students for the opportunity to engage in archery, mountain biking, and slacklining in PE. Students who had not participated in those activities before were "glad" with the chance to "try something new" and to engage in activities that they "always wanted to try" or "don't get to do that often." On the other hand, students who were already familiar with these activities expressed being "excited" that they "got to do in school" activities they already "liked" and felt "confident about it." When asked about their engagement in the new activities, students primary responses were often "it was fun," "I liked it," "it was exciting," or "it was pretty cool."

Students were also asked to identify specific attributes of each activity that made them enjoyable. In regards to archery, numerous participants mentioned that they simply "like shooting a bow." Some students provided more details and described enjoying to "get everything ready," to "be in control," to "concentrate," and to "hear the sound" of the arrow hitting the target. Many students mentioned liking to be constantly challenged to "hit the bulls' eye." Concerning mountain biking, students often recalled "getting outdoors" as their favorite part. Students said that they liked to ride "around the school" and "through the fields," to go "up and down the hills," and to "enjoy the fresh air." One participant said: "I've seen parts of the school outside I've never seen before," while another described the biking experience as "going on an adventure." In regards to slacklining, students frequently reported enjoying to "work on" or "test" their "balance" "while having fun." Many often described slacklining as "thrilling" because it was "challenging" and they had to "focus" and "concentrate on not falling." In addition, students used expressions such as "walking on air," "mission impossible," and "master of concentration" when referring to their enjoyment of slacklining. Other comments related to enjoyment include:

It gave a lot of kids that don't have bikes or a bow to shoot an opportunity. I thought it was nice to see all of the other kids that don't have that stuff smile and happy. $6^{\text {th }} M P A$

I practiced with a bow before but I was excited to do it in class. $7^{\text {th }} \mathrm{LPA}$

It seemed like everybody in gym class had a good time with it. $8^{\text {th }} M P A$ 
It's the adrenaline for me. You get your adrenaline pumping and then you're sitting there holding your breath, pulling back and shooting and then you just let go. $8^{\text {th }}$ LPA

We get stuck in other periods for a long time and then you just get to go outside and feel the breeze and air and the sun on your skin and you just get to have fun out there, be a little kid again. $7^{\text {th }}$ LPA

It's just like pushing your brain to test your balance skills to test so like you've got to just focus and not mess up and try to get to the other side of the line. $8^{\text {th }} \mathrm{LPA}$

\subsubsection{Theme II: Learning}

Across all focus groups students recounted "learning different things" and "new techniques" that ultimately reflected a variety of skills and concepts pertinent to the new activities. Besides learning how to "shoot a bow" students described in detail archery skills developed during the lessons such as "figure out your dominant eye," "lock the bow," and "aim." In respect to mountain biking, participants mentioned learning to "change gears," to ride "uphill and downhill," and to "control the bike in different terrains". Concerning slacklining, students often recalled gaining "balance" as an outcome of participating in the lessons. Moreover, students reported learning personal and social responsible behaviors such as "stay safe and wear a helmet," "be careful when there's people around," and "take turns." Some students referred to learning as "getting the hang of it" and expressed being more comfortable to engage in mountain biking, archery, or slacklining as they regarded themselves as more proficient.

Probably just learning how to ride the mountain bike cause I mean I have a regular bike at home but changing the gears I didn't think I would like that, but I do. $6^{\text {th }} M P A$

But after a while getting to learn it, it really got much better and a lot more students had really focused on what the teacher had taught us. $7^{\text {th }}$ LPA

When asked to describe their most memorable moment regarding the new units, LPA and MPA students consistently referred to their successful experiences across the new units. It seemed that the activities' inherent components (e.g., "hit the bulls' eye," "get all the way across," "riding the trail") allowed students to assess their own performance and develop a sense of "achievement" as a result of their positive experiences. Some students also associated "competition" with their accomplishments indicating that trying to "outdo the next person" encouraged them to "do better." Further, a few girls in both LPA and MPA groups said that archery was a great opportunity to "show up the boys" and "prove them wrong" in regards to their ability to "shoot bows." Interestingly, LPA students mentioned successful experiences more often than MPA students. Some of their comments alluded to "feeling proud," being "more confident to do it outside of school," "showing people what you can do," and not being "a failure anymore."

I thought the satisfaction of knowing how well my balance was. $6^{\text {th }}$ LPA

The fact that I was okay at it, I found something I was okay at. $8^{\text {th }} \mathrm{LPA}$

I think it's put me more in shape than what I used to be cause I used to not be confident in my body shape and what was going on. Now that I've done this every single day it has made me more confident in my appearance. $\sigma^{\text {th }} L P A$

Students also referred to the social interactions that happened during the lessons as beneficial to their learning. According to participants, their PE teachers were very supportive as they "described what to do and how to do it," "showed" and "corrected" them, helped them "to figure it out," and made them feel "safer." In addition, students enjoyed participating in activities that allowed them to interact with and be supported by their peers. They mentioned appreciating to "hang out," "compete with," "learn from," "talk to," "ride with," "cheer on," and "laugh" with their "friends." Many students also identified a visit made by slacklining professionals to the school as their most memorable moment within the three units. Students explained that the professionals "motivated" their "bodies" and made them "determined to try and learn." They also found it "cool" to have "experts" sharing their "experiences" and helping them "to do it right."

I mean they'll [friends], let me see how to put it, they'll help you, like they'll cheer you on if you don't know how to do it. Like if you can't pull it back if you're weak they'll cheer you on and then you can do it. $7^{\text {th }} L P A$

Like that dude that was here he was like talking about how he does like competitions and goes up against people and I was like yeah that would be cool. $8^{\text {th }}$ MPA

\subsubsection{Theme III: Value}

Students indicated that they found value and meaning in the CGRC. They valued the CGRC because those are activities that they could engage in with their family outside of school, in the environment that they live. Students' enthusiasm with mountain biking related to the abundance of "trails," "parks," and "paths" existent in their community and "around" their "houses." One student said: "I think it was fun cause at my house I like to ride bikes up in the 
mountains." On the other hand, data showed that participants valued the inclusion of archery in PE due to its association with hunting, which is a longstanding tradition in the Appalachian community where the CHOICES project was implemented. Students' remarks about having archery in PE referred to archery as "another experience with hunting" and as a "good practice before the hunting season." Likewise, some students described "shooting bows" as an "important life skill" and "useful for the future." One girl said that engaging in archery made her feel "a part of something" while a boy mentioned liking to have his "arm scratched by the bow's string" because it was a sign that he had "actually shot bows." Students' comments alluding to the engagement in the new activities outside of school with their families included: "practice with my grandpa," "I showed my dad," "I go with my mom every week," "me and my sister usually ride out there," and "now that I learned I could ride with them." Others said:

Yeah because my grand mom... she's getting me a mountain bike for Christmas and then that summer, like the first few days of summer she's going to take me to this place where you go mountain biking. So I was like, it's kind of preparing me for the future. $6^{\text {th }} L P A$

I thought like the people that don't know how, they just learned how that you could... this might be handy when they grow up. Maybe they want to be a hunter. $7^{\text {th }}$ LPA

I'm a country person so I don't know I just like doing stuff like that. I think it's just fun to me. $8^{\text {th }} M P A$

Students were also asked if the CGRC influenced their thoughts about PE. They often compared the new activities with traditional forms of PE characterized by them as "common sports," "basketball, kickball, and dodge ball," "stretches and running," "the same routine," "pushups," "tag games," and "a ball coming towards you." They portrayed their previous experiences in PE as "boring," "repetitive," and "not fun." In contrast, students described the CGRC as a "new experience" and "a good change" that made PE "more interesting" and "a lot funner." Students commented that they would "look forward" to going to PE because they could "do something different," "exercise in a fun way," and "learn new things and stuff that can help you long ways."

I liked the idea of getting new activities in gym because a lot of basic gym classes we do just like volleyball, basketball and stuff like that and getting new stuff and keeping people interested in gym class and getting their physical activity is important. $8^{\text {th }}$ LPA

Well, normally in PE we don't really do... On this we actually focus on it and actually try to achieve something instead of just going out and doing whatever. $8^{\text {th }} M P A$

They all have a physical activity, like mountain biking helps your legs; archery helps your strength for your arms, and balance. $6^{\text {th }} M P A$

As a result of this new perspective about PE, participants recurrently called for "more opportunities" to engage. They asked for "more practice" during the lessons expecting that it would help them to "get better at" the activities and "really get into it." According to the students, "40 minutes PE classes" combined with the safety protocols pertaining to archery (i.e., waiting until all students had shot to retrieve arrows), slacklining (i.e., only one students is allowed to travel across the line at a time), and mountain biking (i.e., bike checks and helmets) contributed to decreased time in learning experiences, which was frustrating to them. Participants suggested that the units should be "longer" and occur "more often" so they could engage deeply with the content.

$$
\begin{aligned}
& \text {... if they did it like at the end of every month that'd be so much better because we could then like get more } \\
& \text { practice. } 6^{\text {th }} \text { LPA } \\
& \text { I would love for it to be an everyday thing, or most everyday, like a weekly thing. } 7^{\text {th }} \text { LPA }
\end{aligned}
$$

\subsubsection{Theme IV: Challenges}

When asked to describe aspects of the new units that were challenging, students referred to safety, equipment differentiation, and uncomfortable experiences. Students reported having apprehensive thoughts related to the safety of archery, mountain biking, and slacklining when they first heard they would participate in those activities. Their remarks alluded to the risks inherent to each one of the activities including: "somebody is going to wreck," "I could shoot somebody," and "I thought I was going to fall on my face." Students described being "afraid" and "scared" with the chance of getting injured or injuring someone else while "shooting bows," "wrecking" their bikes, or "falling off" the slackline. Such apprehensive thoughts seemed to be associated with lack of previous experiences, low perceptions of athletic competence, and lack of trust in their peers. Subsequent comments about their engagement in the units indicated that safety concerns were no longer an issue given the safety protocols established by their teachers.

I kept saying, I am going to die because I'm not very balanced. $7^{\text {th }} L P A$

First of all, some of those people in there they kind of scare me. So I was like he's going to trust us with bows and arrows? I was like what's wrong? I was like we can do this? $8^{\text {th }}$ MPA

I felt safer cause the teachers described what to do and how to do it. $8^{\text {th }}$ MPA 
Students were also able to identify potential modifications to the equipment that could have helped them to perform better in all three activities. According to the students varied "bow sizes" and "strengths," "wider" and "shorter" slacklines, and "different bike sizes" could have facilitated their learning.

Maybe they could've also started us off like before we actually got on the actual rope we could've like started trying to practice on like the lines of the gym or something. $6^{\text {th }}$ LPA

It was just trying to climb up on the bike cause the bikes are bigger than your normal size bike. $8^{\text {th }}$ LPA

Finally, students mentioned uncomfortable experiences that made the activities challenging. Students did not like to have the bowstring "scraping" or "burning" their arms. Similarly, despite the high quality bikes provided students often complained about the "bike seats" and claimed that they "hurt." "Falling" from the slackline seemed to be uncomfortable to many students, but data showed that even "falling many times" did not prevent them to continue trying nor to enjoy the activity.

Yeah, where the string smacks the side of your arm that hurts. $7^{\text {th }} L P A$

Yes, you went on it and went up and down and your butt would be hurting on that seat. The seat was not very comfortable. $7^{\text {th }}$ LPA

Although both LPA and MPA students commented on the challenges related to the CGRC, the frequency of these comments was two times higher among LPA students. This suggests that LPA students were more sensitive to the challenges they encountered as they learned archery, mountain biking, and slacklining in PE.

\section{Discussion}

The quality of experiences in PE might be one of the most important influences on children and adolescents' participation in PA outside of the school setting (Ennis, 2011; MacPhail, 2011). Accordingly, scholars have recommended that PE curricula reform is key in our efforts to increase children and adolescents' engagement in and appreciation for physically active lifestyles (Bulger \& Housner, 2009; Ennis, 2014; Kinchin \& O'Sullivan, 2003). This study investigated how middle school students perceived the inclusion of CGRC in a newly developed PE curriculum of an Appalachian middle school. Findings reveled that archery, mountain biking, and slacklining lessons allowed LPA and MPA students to voice positive answers to the questions Am I able? and Is it worth it? outlined in the YPAP model as predisposing factors of PA (Welk, 1999).

\subsection{Am I able?}

Children's perceptions of competence regarding their ability to perform in sports and PAs are critical for the establishment of positive attitudes towards physically active lifestyles (Ennis, 2011; Woods, Bolton, Graber, \& Crull, 2007). Offering young people access to programs, facilities, and equipment is the first step in fostering the development of competencies needed to engage in sports and PAs (Welk, 1999). In this study, LPA and MPA participants often used the word "opportunity" to depict their experiences with the CGRC indicating that they appreciated having access to activities that otherwise would not be offered to them in the school setting. Because PE classes might be the only instance where many young people have access to PA, it is important that PE curricula strive to broaden students' PA experiences, rather than be limited to selected team sports (Ferry \& McCaughtry, 2013). Maintaining team sports as the PE status quo is likely to continue reinforcing the alienation of those students who might have other interests and abilities from active lifestyles. One LPA participant voiced this issue as he commented about his experience in archery: "I finally found something I was ok at." This statement highlights the need to include alternative forms of PA in the curriculum so that children can identify activities in which they are successful at and attracted to (Prusak et al., 2014; Tanehill et al., 2015).

Favorable environmental conditions can also contribute to children and adolescents' development of positive perceptions of competence in PE (Bernstein et al., 2011). One of the critical impediments for quality PE is the inadequacy of school's environmental conditions such as equipment shortage, large class sizes, and inadequate facilities (Bulger \& Housner, 2009; Prusak et al., 2010). The investment made through the PEP grant on equipment purchase and maintenance, (i.e., bikes, helmets, bows, arrow, slacklines) and teachers' continuous professional development was essential for the establishment of the CGRC in the curriculum. As a result, teachers were able to create a safe, inclusive, developmentally appropriate, and authentic environment that fostered students' successful performance and consequent positive perceptions of competence in all three units. An interesting finding was the higher frequency to which LPA students alluded to possible equipment modifications that could facilitate skill development. These students were aware of their individual needs and found important to voice them and request specific accommodations. In addition, students were concerned with safety and more prone to participate when a safe environment was established by the teachers.

Nevertheless, granting youth access to varied movement experiences and establishing adequate learning environments does not determine or guarantee the development of positive perceptions of competence regarding engagement in sports and PA. In order for students to answer yes to the question Am I able? they should be provided with ample and developmentally appropriate opportunities for skill development. One of the strongest criticisms to multi-activity approaches to PE is the focus on full-sided game play and the lack of skill development opportunities (Kirk, 2006), 
which contributes to the dominance of highly skilled students and the reinforcement of low perceptions of competence among students who are not as skilled (Ennis, 2014; Garn et al., 2011). Participants in this study vividly recalled learning new concepts and skills as well as being successful across the three new units. Remarkably, LPA students mentioned successful experiences almost twice as much as MPA students suggesting that they emphasized the importance of skill development and were thrilled about being able to successfully participate in PE. It appeared that archery, mountain biking, and slacklining leveled the playing field among all students, which increased their chances to succeed while competing against self and others (e.g., girls wining over boys).

\subsection{Is it worth it?}

In the YPAP model, outcome expectancy of PA is operationalized as enjoyment from and attraction to PA (Welk \& Schaben, 2004). Findings from this study indicated that LPA and MPA students voiced enjoying and being attracted to the CGRC, which resulted in a renewed perspective of PE. The most frequent term used by students to describe the units was "fun," which was then associated with novelty, success, social interactions, the outdoor environment, challenge, competition, and specific attributes of each activity (e.g., hitting the bulls' eye). Moreover, enjoyment of archery, mountain biking, and slacklining generated a new and positive perspective of PE amongst the majority of students. They compared this approach to past experiences based on exercise routines, and team sports and indicated enjoyment as the main reason for a renewed value of PE. Recently, scholars have suggested that one way to attract children and adolescents into active lifestyles is to provide them with meaningful movement experiences (Enright \& O’Sullivan, 2012; Kretchmar, 2000; McCaughtry, 2009). Kretchmar (2000) suggested that PE should strive to promote personal and shared meaning through the inclusion of content that resonates with students' individual and sociocultural values. At the individual level, students from both groups found value and purpose in the new units and mentioned that learning mountain biking, archery, and slacklining was beneficial for leisure pursuits, fitness enhancement, personal challenge, and skill acquisition. At the sociocultural level, students indicated that it is worth to be physically active when they can play with their peers, witness others perform efficiently, receive adequate support from their teachers, and learn activities that are relevant for their culture and they can engage in with their family outside of school, in the environment that they live.

Consistent with other studies' reports, the opportunity to socialize with friends during PE was described by LPA and MPA students as a facilitator to engagement and learning (Prusak et al., 2014; Tanehill et al., 2015). The paradox here is the fact that students felt more connected with their peers in individual activities than in team sports. Students mentioned enjoying watching others perform and receiving emotional support from those around them, confirming the reinforcing role of peers in PA participation (Davison, 2004). Similarly, students referred to their teachers as positive influences in their experiences with the new content. In this study teachers were key participants in the content selection, units design, and lesson implementation, resulting in positive learning experiences for all students rather than a selected minority. This happened as a result of providing continuous professional development for teachers with the purpose of empowering them in the decision-making process regarding curriculum development and implementation.

Finally, students communicated valuing archery, mountain biking, and slacklining because those activities could connect them with their families, their natural environment, and their culture. For example, archery strongly resonated with both LPA and MPA students' sociocultural background given its relation to hunting, a traditional practice in the Appalachian community in which this study was conducted. Students were thrilled to replicate in school a practice that so closely relates to their way of life. Kretchmar (2000) proposed that "the cultural connotations of movement remind individuals who they are (a family, a tribe, a nation), who they belong to (their parents, the values and truths of their time), and where they came from (their particular traditions and history)" (p.5). Students often mentioned that it is worth it to learn activities they can engage in with their family in the environment in which they live. Students voiced that those activities generated a sense of belongingness to their environment and culture by allowing them to learn things they perceive as meaningful in their contexts outside of school. These are important findings that echo the notion that students' individual and sociocultural values must be integrated in the curriculum, thus opening room for meaningful movement experiences to take place in PE (Enright \& O'Sullivan, 2012; Kretchmar, 2000; MacPhail, 2011; McCaughtry, 2009).

In the YPAP model, the questions Am I able? and Is it worth it? are interrelated, suggesting that perceptions of competence and outcomes expectancy mutually influence one another. According to Welk (1999) "children value what they are good at doing and pursue things they value" (p. 13). In this study, participants often alluded to this relationship. They enjoyed the units because they were successful, but they were also motivated to practice because the activities were fun. Likewise, they valued the units because they learned new skills, and they were eager to learn new skills because they found meaning in the activities. This constant interaction between perceived competence and outcomes expectancy has also been described in other studies examining students' perceptions of PE from a qualitative standpoint (Bernstein et al., 2001; Prusak et al., 2014; Tanehill et al., 2015). Hence, it is vital that PE learning experiences are purposeful designed to reinforce the dialectic relationship between these constructs.

\section{Conclusion, Limitations, and Future Directions}

Findings from this study concur with recommendations for the design of PE curricula that resonate with students' interests, sociocultural background, and geographical environment. Such task calls the attention of physical educators to the kinds of movement experiences that children and adolescents find enjoyable, are successful in, and perceive as 
meaningful. By placing students in the center of curriculum design, implementation, and evaluation, physical educators can continue to learn from their voices and provide them with experiences in sports and PAs that are relevant and can be sustained for a lifetime. In this study, participant LPA and MPA students demonstrated similar perceptions and responses to the inclusion of archery, mountain biking, and slacklining in the curriculum and indicated valuing: (a) opportunities for skill development, (b) successful experiences (c) peer interactions, (d) the outdoor environment, (e) competition, (f) adequate equipment, (g) positive role models (h) new, challenging, and fun movement and experiences, and (i) activities that connect them with their family, culture, and geographical environment. In addition, data revealed that the new content contributed to positive perceptions of competence and a renewed perspective of PE among LPA and MPA students. These findings support that innovative PE curriculum models have the potential to attract middle school-aged children into active lifestyles, particularly those who are less predisposed to be physically active. This study was limited to the extent to which students provided socially desirable answers since data was collected during PE classes and students' perceptions about selected units and not the entire curriculum. Future studies on children and adolescents' perceptions of CGRC are encouraged. Longitudinal studies with the purpose of identifying changes in students' perceptions and responses over time should be considered. Also, research examining students' reactions to CGRC in urban schools might provide another perspective on innovative approaches to PE.

\section{References}

Baker, B. L., \& Davison, K. K. (2011). I know I can: A longitudinal examination of precursors and outcomes of perceived athletic competence among adolescent girls. Journal of Physical Activity \& Health, 8(2), 192-199.

Bernstein, E., Phillips, S. R., \& Silverman, S. (2011). Attitudes and perceptions of middle school students toward competitive activities in physical education. Journal of Teaching in Physical Education, 30(1), 69-83.

Brustad, R. (1993). Who will go out and play? Parental and psychological influences on children's attraction to physical activity. Pediatric Exercise Science, 5(3), 210-223.

Brustad, R. J. (1996). Attraction to physical activity in urban school children: Parental socialization and gender influences. Research Quarterly for Exercise and Sport, 67(3), 316-323. doi:10.1080/02701367.1996.10607959

Bulger, S. M., \& Housner, L. D. (2009). Relocating from easy street: Strategies for moving physical education forward. Quest (00336297), 61(4), 442-469.

Centers for Disease Control and Prevention. (2006). Physical Education Curriculum Analysis Tool. Atlanta, GA: Author

Darbyshire, P., MacDougall, C., \& Schiller, W. (2005). Multiple methods in qualitative research with children: More insight or just more? Qualitative Research, 5(4), 417-436. doi:10.1177/1468794105056921

Davison, K. K. (2004). Activity-related support from parents, peers and siblings and adolescents' physical activity: Are there gender differences? Journal of Physical Activity \& Health, 1(4), 363.

Ennis, C. D. (2006). Curriculum: Forming and reshaping the vision of physical education in a high need, low demand world of schools. Quest (00336297), 58(1), 41-59.

Ennis, C. D. (2011). Physical education curriculum priorities: Evidence for education and skillfulness. Quest (00336297), 63(1), 5-18.

Ennis, C. D. (2014). What goes around comes around ... or does it? Disrupting the cycle of traditional, sport-based physical education. Kinesiology Review, 3(1), 63-70.

Enright, E., \& O’Sullivan, M. (2012). Physical education “in all sorts of corners.” Research Quarterly for Exercise and Sport, 83(2), 255-267. http://doi.org/10.1080/02701367.2012.10599856

Enright, E., \& O’Sullivan, M. (2013). “Now, I'm magazine detective the whole time”: Listening and responding to young people's complex experiences of popular physical culture. Journal of Teaching in Physical Education, 32(4), $394-418$.

Ferry, M., \& McCaughtry, N. (2013). Secondary physical educators and sport content: A love affair. Journal of Teaching in Physical Education, 32(4), 375-393.

Garn, A. C., Cothran, D. J., \& Jenkins, J. M. (2011). A qualitative analysis of individual interest in middle school physical education: Perspectives of early-adolescents. Physical Education \& Sport Pedagogy, 16(3), 223-236.

Glaser, B.G., \& Strauss, A.L. (1967). The discovery of grounded theory: Strategies for qualitative research. New York: Aldine.

Harter, S. (2012) Self-Perception Profile for Children: Manual and questionnaires (grades 3-8) [Revision of the Selfprofile for Children, 1985]. University of Denver, Retrieved from http://portfolio.du.edu/SusanHarter/page/44210

Kinchin, G. D., \& O’Sullivan, M. (2003). Incidences of student support for and resistance to a curricular innovation in high school physical education. Journal of Teaching in Physical Education, 22(3), 245-260.

Kirk, D. (2006). Sport education, critical pedagogy, and learning theory: Toward an intrinsic justification for physical education and youth sport. Quest (00336297), 58(2), 255-264. 
Kretchmar, R. S. (2000). Movement subcultures: Sites for meaning. Journal of Physical Education, Recreation \& Dance, 71(5), 19-25.

Krueger, R. A., \& Casey, A. M., (2009). Focus groups: A practical guide for applied research. Thousand Oaks, CA: Sage Publications.

MacPhail, A. (2011) Youth voices in PE and sport: What are they telling us?. In Armour, K. (Ed.) Sport pedagogy: An introduction for teaching and coaching (pp.105-116). New York, NY: Routledge.

McCaughtry, N. (2009). The child and the curriculum: Implications of Deweyan Philosophy in the pursuit of "cool" physical education for children. In L. Housner, M. Metzler, P. Schempp, \& T. Templin (Eds.), Historic traditions and future directions of research on teaching and teacher education. Morgantown, WV: Fitness Information Technology.

Marshall, C., \& Rossman, G. B. (2006). Designing qualitative research. Thousand Oaks, CA: Sage Publications.

Miles, M., B., \& Huberman, A., M. (1994). Qualitative data analysis. Thousand Oaks, CA: Sage Publications.

Muris, P., Meesters, C., \& Fijen, P. (2003). The Self-Perception Profile for Children: Further evidence for its factor structure, reliability, and validity. Personality and Individual Differences, 35(8), 1791-1802. doi:10.1016/S01918869(03)00004-7

Oliver, K. L., \& Oesterreich, H. A. (2012). Student-centered inquiry as curriculum as a model for field-based teacher education. Journal of Curriculum Studies, 45(3), 394-417. http://doi.org/10.1080/00220272.2012.719550

Paxton, R. J., Estabrooks, P. A., \& Dzewaltowski, D. (2004). Attraction to physical activity mediates the relationship between perceived competence and physical activity in youth. Research Quarterly for Exercise and Sport, 75(1), 107111. doi:10.1080/02701367.2004.10609139

Prusak, K. A., Davis, T., Pennington, T. R., \& Wilkinson, C. (2014). Children's perceptions of a district-wide physical education program. Journal of Teaching in Physical Education, 33(1), 4-27. doi:10.1123/jtpe.2013-0004

Prusak, K. A., Pennington, T., Graser, S. V., Beighle, A., \& Morgan, C. F. (2010). Systemic success in physical education: The East Valley phenomenon. Journal of Teaching in Physical Education, 29(1), 85-106.

Society of Health and Physical Educators. (2009). Opportunity to learn: Guidelines fro middle school physical education. Reston, VA: Author

Society of Health and Physical Educators. (2014). National standards \& grade-level outcomes for $k$-12 physical education. Reston, VA: Author

Society of Health and Physical Educators. (2015). The essential components of physical education [Guidance Document]. Reston, VA: Author.

Tannehill, D., MacPhail, A., Walsh, J., \& Woods, C. (2015). What young people say about physical activity: the Children's Sport Participation and Physical Activity (CSPPA) study. Sport, Education \& Society, 20(4), 442-462.

Tannehill, D., van der Mars, H., \& MacPhail, A. (2015). Building effective physical education programs. Burlington, MA: Jones \& Bartlett Learning

Wallhead, T. 1., \& Buckworth, J. (2004). The role of physical education in the promotion of youth physical activity. Quest (00336297), 56(3), 285-301.

Welk, G. J., \& Schaben, J. A. (2004). Psychosocial correlates of physical activity in children: A study of relationships when children have similar opportunities to be active. Measurement in Physical Education and Exercise Science, 8(2), 63-81.

Woods, A. M., Bolton, K. N., Graber, K. C., \& Crull, G. S. (2007). Chapter 5: Influences of perceived motor competence and motives on children's physical activity. Journal of Teaching in Physical Education, 26(4), 390-403

\section{Notes}

Note 1. Figure 1: From "The youth physical activity promotion model: A conceptual bridge between theory and practice”, by G. Welk, 1999, Quest, 51(1), p. 12. Copyright 1999 by Taylor \& Francis LLC. Reprinted with permission. Note2. The reference at the end of each quote indicates: grade $\left(6^{\text {th }}, 7^{\text {th }}\right.$, or $\left.8^{\text {th }}\right)$ and group (LPA or MPA). 